\title{
Assessment of patient safety and the efficiency of facility utilization following simplified ultra-rapid intravenous infusion of hepatitis $B$ immunoglobulin in a high-volume liver transplantation center
}

\author{
I-Ji Jeong', Shin Hwang ${ }^{1,2}$, Dong-Hwan Jung ${ }^{1}$, Gi-Won Song ${ }^{1}$, Gil-Chun Park', Chul-Soo Ahn ${ }^{1}$, \\ Deok-Bog Moon ${ }^{1}$, Ki-Hun Kim ${ }^{1}$, Tae-Yong Ha ${ }^{1}$, Hea-Seon Ha ${ }^{2}$, Jung-Ja Hong ${ }^{2}$, In-Ok Kim², and Sung-Gyu Lee ${ }^{1}$

\begin{abstract}
${ }^{1}$ Division of Hepatobiliary Surgery and Liver Transplantation, Department of Surgery, Asan Medical Center, University of Ulsan College of Medicine, ${ }^{2}$ Organ Transplantation Center, Asan Medical Center, Seoul, Korea
\end{abstract}

\begin{abstract}
Backgrounds/Aims: This study intended to evaluate patient safety and efficiency of facility utilization following simplified ultra-rapid intravenous infusion of hepatitis B immunoglobulin (HBIG) in recipients of hepatitis B virus-associated adult liver transplantation (LT), who visited our outpatient clinic. Methods: Our simplified ultra-rapid infusion protocol was to directly infuse $50 \mathrm{ml}$ volume of 10,000 IU HBIG for 20-25 minutes on an ambulatory basis. The incidence of adverse side-effects and the efficiency of facility utilization were assessed retrospectively. Results: A total of 1,513 patients received 12,472 sessions of HBIG infusion according to simplified ultra-rapid infusion method. Of these, 1,172 patients were converted from conventional ultra-rapid infusion method, and received 8,352 sessions of HBIG infusion for 18 months (mean 7.1 times; 4.8 times per year). The remaining 341 de novo patients received 4,120 sessions of HBIG infusion for 18 months (mean 12.1 times; 8.1 times per year). None of these patients experienced any adverse side-effects following the simplified ultra-rapid infusion. The maximal capacity of HBIG infusion sessions at the injection facility of our outpatient clinic was increased from 65-70 sessions to 80 sessions, after introduction of simplified ultra-rapid infusion method. Mean trough anti-HBs titer was lower, and mean interval of HBIG infusion was longer in the combination therapy group compared with HBIG monotherapy group. Conclusions: Our high-volume study indicates that in nearly all LT recipients, rapid infusion of highly purified HBIG within 30 minutes was well-tolerated. This suggests that it would be reasonable to perform simplified ultra-rapid infusion protocol widely for patient convenience. (Ann Hepatobiliary Pancreat Surg 2019;23:128-132)
\end{abstract}

Key Words: Hepatitis B immunoglobulin; Hepatitis B virus; Prophylaxis; Liver transplantation

\section{INTRODUCTION}

Prevention of recurrent exposure to hepatitis B virus (HBV) is essential after liver transplantation (LT) in patients infected with HBV. Many protocols for HBV prophylaxis after LT include the administration of hepatitis B immunoglobulin (HBIG). ${ }^{1-4}$ HBIG treatment has been used for a long time because of its effectiveness, but it has major drawbacks including high cost, resistant viral mutation, and a cumbersome injection process. ${ }^{1,2,5}$ However, the route of HBIG administration has evolved to improve patient compliance.

In Korea, HBIG agents were initially developed for in- tramuscular injection in small doses. Thus long infusion periods of up to 4-8 hours were required for the administration of 10,000 IU HBIG in order to prevent adverse events. Following the development of more purified HBIG agents suitable for intravenous injection, the infusion time was successfully shortened to 1-2 hours, and with increased experience, we adopted an ultra-rapid infusion for 30 minutes. $^{6}$ This shortened duration of infusion allowed us to utilize the hospital facility more effectively and enabled us to organize patient-friendly HBIG infusion schedules.

However, a progressive increase in LT recipients requiring regular HBIG infusions forced us to further mod-

Received: March 7, 2019; Revised: March 12, 2019; Accepted: March 15, 2019

Corresponding author: Shin Hwang

Department of Surgery, Asan Medical Center, University of Ulsan College of Medicine, 88 Olympic-ro 43-gil, Songpa-gu, Seoul 05505, Korea Tel: +82-2-3010-3930, Fax: +82-2-3010-6701, E-mail: shwang@amc.seoul.kr

Copyright (C) 2019 by The Korean Association of Hepato-Biliary-Pancreatic Surgery

This is an Open Access article distributed under the terms of the Creative Commons Attribution Non-Commercial License (http://creativecommons.org/ licenses/by-nc/4.0) which permits unrestricted non-commercial use, distribution, and reproduction in any medium, provided the original work is properly cited. Annals of Hepato-Biliary-Pancreatic Surgery • pISSN: 2508-5778 - eISSN: 2508-5859 
ify the HBIG infusion protocol to be more efficient in terms of time and effort. In order to reduce the infusion time as well as the patients' wait time, and to improve the efficiency of our facility, we evaluated the outcome of simplified ultra-rapid HBIG infusion protocol administered to $\mathrm{HBV}$-associated adult $\mathrm{LT}$ recipients visiting the outpatient clinic.

\section{PATIENTS AND METHODS}

This single-arm study intended to evaluate the patient safety and the efficiency of facility utilization following simplified ultra-rapid intravenous infusion of HBIG in HBV-associated adult LT recipients who visited our outpatient clinic.

At our institution, 10,000 IU HBIG was infused during the anhepatic phase of LT operation, daily during the first week, once weekly for the next 1 month, and once monthly after that. After the first year of LT, the interval period between regular HBIG infusions was gradually increased to 8-16 weeks on a patient-by-patient basis, in order to maintain a trough serum antibody to hepatitis B surface antigen (anti-HBs) titer $\geq 500 \mathrm{IU} / \mathrm{L}$. A high-genetic barrier nucleos(t)ide analog (entecavir or tenofovir) has often been added as combination therapy, especially in LT recipients showing rapid HBIG consumption or risk of hepatocellular carcinoma recurrence. ${ }^{7,8}$

We previously presented the protocol for conventional ultra-rapid HBIG infusion, ${ }^{6}$ in which $50 \mathrm{ml}$ volume of 10,000 IU HBIG (collection of 5 bottles of $10 \mathrm{ml} \mathrm{HBIG}$ 2,000 IU without dilution) was continuously infused without using an infusion pump for 25 minutes, followed by a 5-minute wash-out of the infusion line with $50 \mathrm{ml} \mathrm{5 \%}$ dextrose water. To ensure safety, this ultra-rapid infusion was performed in patients on bed-rest. From March 2010 to September 2016, more than 30,000 sessions of ultrarapid $\mathrm{HBIG}$ infusion were performed in more than 2,500 LT recipients.

In our simplified ultra-rapid infusion method, two points were modified as follows: wash-out of the infusion line was omitted, and sitting/ambulation was permitted during the HBIG infusion. Thus, $50 \mathrm{ml}$ volume of 10,000 IU HBIG (prefilled at the institutional pharmacy through collection of 5 bottles of $10 \mathrm{ml} \mathrm{HBIG} \mathrm{2,000} \mathrm{IU)} \mathrm{was} \mathrm{directly} \mathrm{infused}$ for 20-25 minutes on an ambulatory basis.
After a preliminary evaluation for 3 months with alternating administration of the conventional and simplified infusion methods, since January 2017 the simplified method replaced the conventional method.

We retrospectively analyzed the medical records of adult LT recipients who were administered HBIG regularly at the outpatient clinic during the 18-month study period from January 2017 to July 2018. Patients who were administered HBIG alternatively at other institutions were excluded. Apart from that, five patients who were administered HBIG slowly for 2 hours due to a past history of HBIG-associated urticaria were also not included in this study. All adverse side-effects were recorded in the HBIG infusion sheet.

Continuous variables were reported as mean \pm standard deviation. $p$-value $<0.05$ was considered statistically significant. Statistical analyses were performed using SPSS (version 22; IBM, New York, NY). The study protocol was approved by the institutional review board of the Asan Medical Center (2018-0739).

\section{RESULTS}

\section{Patient safety}

The profiles of adult LT recipients enrolled in this study are summarized at Table 1. A total of 1,513 patients received 12,472 sessions of HBIG infusion according to the simplified ultra-rapid infusion protocol.

Of them, 1172 patients were converted from the conventional ultra-rapid infusion method, and received 8,352 sessions of HBIG infusion for a duration of 18 months (mean 7.1 times; 4.8 times per year). None of them experienced any adverse side-effects following the simplified ultra-rapid infusion.

The remaining 341 patients, who were de novo patients, were provided the simplified ultra-rapid infusion method at the outpatient clinic, although they had received a similar ultra-rapid infusion during their hospital stay during the early post-transplant period. These de novo patients received HBIG infusion in 4,120 sessions over 18 months (mean 12.1 times; 8.1 times per year), and none of them experienced any adverse side-effects following the simplified ultra-rapid infusion. 
Table 1. Profile of 1,513 patients who were administered HBIG at the outpatient clinic during the study period

\begin{tabular}{lc}
\hline \multicolumn{1}{c}{ Parameters } & Values \\
\hline Age at LT (year) & $51.6 \pm 8.8$ \\
Sex (n) & $1,157(76.5 \%)$ \\
Male & $356(23.5 \%)$ \\
Female & \\
Primary diseases (n) & $1,452(96.0 \%)$ \\
HBV-associated LC & $61(4.0 \%)$ \\
HBV-associated ACLF & $1,513(100 \%)$ \\
Pre-transplant HBsAg positivity (n) & $602(39.8 \%)$ \\
Pre-transplant HBeAg positivity (n) & $19.9 \pm 9.7$ \\
MELD score & $642(42.4 \%)$ \\
Concurrent HCC (n) & $908(60.0 \%)$ \\
Pre-transplant use of antiviral agent (n) \\
Yes \\
No & $605(40.0 \%)$ \\
Pre-transplant HBV DNA status & \\
Undetectable & $423(28.0 \%)$ \\
$\leq 4 l o g$ IU/ml & $559(36.9 \%)$ \\
>4log IU/ml & $531(35.1 \%)$ \\
Type of LT (n) & \\
Living donor LT & $1,391(91.9 \%)$ \\
Deceased donor LT & $122(8.1 \%)$ \\
Type of HBV prophylaxis (n) & \\
HBIG-antiviral agent combination therapy & $892(59.0 \%)$ \\
HBIG monotherapy & $621(41.0 \%)$ \\
\hline
\end{tabular}

HBIG, hepatitis B immunoglobulin; ACLF, acute-on-chronic liver failure; HBV, hepatitis B virus; $\mathrm{HCC}$, hepatocellular carcinoma; LC, liver cirrhosis; LT, liver transplantation; MELD, model for end-stage liver disease; HBsAg, HBV surface antigen; $\mathrm{HBeAg}, \mathrm{HBV}$ e antigen

\section{Efficiency of facility utilization}

The injection facility of our outpatient clinic had a maximal capacity of 65-70 HBIG infusion sessions per day until 2016. There were many other LT recipients at our institution who were not included in the study, thus more than 60 sessions of HBIG infusions had been performed per day until 2016. Considering that the patients were not evenly distributed along the day, the injection facility was often overcrowded.

Our simplified modification reduced the infusion time as well as workload by approximately $15 \%$, which indicates that the turnover rate of HBIG infusion was also increased by $10-15 \%$ without expansion of the injection facility. Since 2017, the maximal capacity of HBIG infusion sessions increased to 80 per day. The improvement in turnover rate also led to a shortening of the waiting period at the injection facility from 15 minutes to 5-10

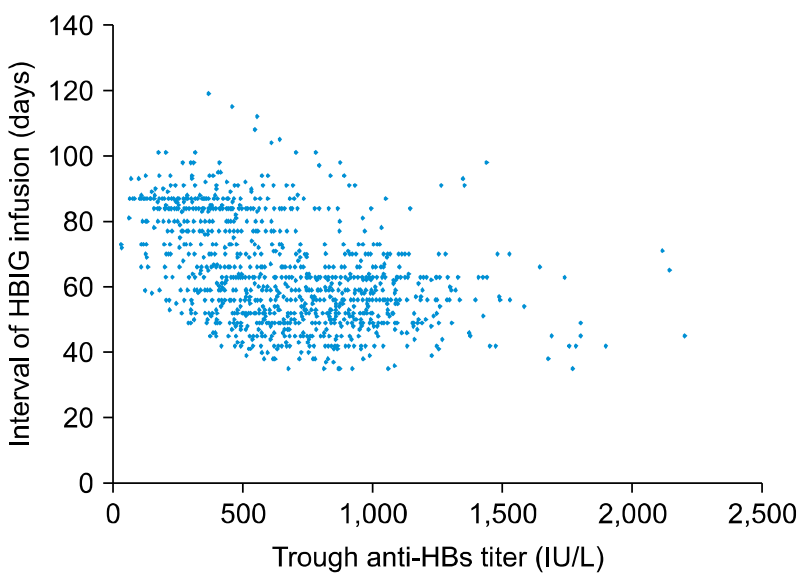

Fig. 1. Scatter plot showing the association between the trough anti-HBs titer and hepatitis B immunoglobulin (HBIG) infusion intervals in 1,172 patients converted from the conventional ultra-rapid infusion method to simplified ultra-rapid infusion method.

minutes. Thus, it became easier to schedule the HBIG injection appointments at the outpatient clinic, leading to increased patient compliance.

\section{Cross-sectional distribution of trough anti- HBs titer}

Correlation between the intervals of HBIG infusion and trough anti-HBs titer at the last measurement is depicted in Fig. 1. The mean trough anti-HBs titer and the mean interval of HBIG infusion were $619.1 \pm 301.1 \mathrm{IU} / \mathrm{L}$ and $66.4 \pm 15.8$ days, respectively in the HBIG-antiviral combi-

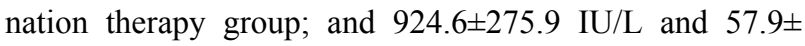
12.4 days, respectively in the HBIG monotherapy group $(p<0.001$ for anti-HBs titer and $<0.001$ for infusion interval). Considering that the target trough anti-HBs titer is set at $500 \mathrm{IU} / \mathrm{L}$ at our institution, a majority of patients showed excessively high trough anti-HBs titers.

\section{DISCUSSION}

HBIG infusion is currently used in many transplant centers as a component of combination therapy or monotherapy for post-transplant HBV prophylaxis. ${ }^{1-7}$ In the real-world practice, HBIG infusion is an important component to determine the intervals between outpatient clinic visits because self-administration of HBIG is not yet permissive. Prefilled intramuscular injections containing HBIG of very high concentration, similar to insulin, are currently 
under investigation, though not yet commercially available. Thus, it is necessary for LT patients to visit hospitals regularly in order to receive HBIG.

In order to enhance patient compliance, two factors should be considered. The first is easy patient-friendly administration of HBIG. In practice, patients prefer short appointments to receive HBIG for convenience. This was the primary reason for the introduction of the simplified ultra-rapid infusion method as an improvement in the administration of HBIG. The second is to prolong the intervals between HBIG infusions, making outpatient clinic visits less frequent. Since the efficacy of HBIG monotherapy is not high, an adequate target trough level of anti-HBs was set to prevent HBV recurrence. Antiviral agents are highly effective in suppressing HBV replication, thus it is reasonable to lower the target anti-HBs trough level in patients undergoing a combination therapy of HBIG with an antiviral agent. ${ }^{9-13}$ Our findings regarding the cross-sectional distribution of trough anti-HBs revealed that a majority of our patients were administered HBIG more often than required, indicating that individualized adjustments in the HBIG infusion intervals are necessary.

Our simplified ultra-rapid infusion protocol has proven to be convenient for patients because it reduces the time they spend at the outpatient clinic. Nearly all the patients treated using this protocol were satisfied and preferred it to the conventional infusion method. Occupancy of patient beds was one of the limiting factors for turnover of HBIG infusion in the outpatient clinic. For reasons of safety, although HBIG was infused under bed rest, sitting or ambulation was usually allowed in the patients with no physical problems, and was proven to be safe following numerous sessions of HBIG infusion in this study. At our institution, the number of LT recipients visiting the outpatient clinic has increased progressively, ${ }^{14,15}$ making it difficult to arrange HBIG infusion schedules at their convenience. We believe that our simplification of the ultra-rapid infusion method does not lower patient safety.

There was one significant reason for abandoning the wash-out procedure with $50 \mathrm{ml} \mathrm{5 \%}$ dextrose water in our simplified method. This wash-out procedure was intended to completely infuse the expensive HBIG into the recipient without any amount of the solution remaining in the infusion line. Our studies revealed that the mean amount of HBIG remaining in the infusion line was estimated to be less than $1.5 \mathrm{ml}(<3 \%)$. This was considered to be negligible considering the additional effort required for the wash-out process. In addition, dilution of the HBIG with $5 \%$ dextrose water could produce faint floating substances in some rare instances, which were identified as immunoglobulin G. The Korean Pharmacopoeia suggests that 10,000 IU HBIG should be dripped after mixing with $150 \mathrm{ml}$ of $5 \%$ dextrose water (permitted at December 2006). This protocol was observed in our institution before introduction of the ultra-rapid infusion method in February 2010, but was modified primarily for the purpose of avoiding the infusion pump. Furthermore, dilution or washing with $5 \%$ dextrose water was eliminated from our current simplified protocol. Based on these reasons, we suggest revising the recommendation for HBIG administration described in the Korean Pharmacopoeia.

A new HBIG product containing $50 \mathrm{ml}$ of $10,000 \mathrm{IU}$ HBIG will be available soon in the market in Korea. Considering that regular infusion with a fixed dose of 10,000 IU HBIG has been routinely performed in Korea and collection of bottles with small dose is necessary, such a high-dose bottle agent will be helpful to perform ultra-rapid infusion as well as to prevent collection procedure-related contamination.

This study has some limitation. This study is a highvolume study but performed in a single institution. This study is a retrospective study, thus a prospective study design is necessary for follow-up studies. This study did not include all sessions of HBIG infusion in our institution because data collection was performed on only patient base not infusion session base.

In conclusion, our high-volume study indicated that the rapid infusion of highly purified HBIG within 30 minutes was well tolerated by nearly all LT recipients. This suggests that it would be reasonable to perform the simplified ultra-rapid infusion protocol widely for patient convenience.

\section{ACKNOWLEDGEMENTS}

This study was supported by the Cooperative Research Program from Korean Organ Transplantation Registry (KOTRY) (Grant No. 2018-001). 


\section{REFERENCES}

1. Hwang S, Lee SG, Ahn CS, Kim KH, Moon DB, Ha TY, et al. Prevention of hepatitis B recurrence after living donor liver transplantation: primary high-dose hepatitis B immunoglobulin monotherapy and rescue antiviral therapy. Liver Transpl 2008; 14:770-778.

2. Mohanty SR, Cotler SJ. Management of hepatitis B in liver transplant patients. J Clin Gastroenterol 2005;39:58-63.

3. Terrault N, Roche B, Samuel D. Management of the hepatitis $B$ virus in the liver transplantation setting: a European and an American perspective. Liver Transpl 2005;11:716-732.

4. Roche B, Samuel D. Evolving strategies to prevent HBV recurrence. Liver Transpl 2004;10(10 Suppl 2):S74-S85.

5. Ghany MG, Ayola B, Villamil FG, Gish RG, Rojter S, Vierling $\mathrm{JM}$, et al. Hepatitis B virus $\mathrm{S}$ mutants in liver transplant recipients who were reinfected despite hepatitis B immune globulin prophylaxis. Hepatology 1998;27:213-222.

6. Hwang S, Yu YD, Park GC, Choi YI, Park PJ, Jung SW, et al. Safety of ultra-rapid intravenous infusion of hepatitis B immunoglobulin in liver transplant recipients. Transplant Proc 2011;43:1780-1782.

7. Choi HJ, Kim DG, Kim SI, Wang HJ, Joh JW, Suh KS, et al. A multicenter phase III study to evaluate the efficacy and safety of hepabulin, a new hepatitis B immunoglobulin, in liver transplantation recipients with hepatitis B. Ann Transplant 2017;22: 740-748.

8. Korean Association for the Study of the Liver. KASL clinical practice guidelines: management of chronic hepatitis B. Clin Mol
Hepatol 2016;22:18-75.

9. Roche B, Roque-Afonso AM, Nevens F, Samuel D. Rational basis for optimizing short and long-term hepatitis B virus prophylaxis post liver transplantation: role of hepatitis B immune globulin. Transplantation 2015;99:1321-1334.

10. Gane EJ, Angus PW, Strasser S, Crawford DH, Ring J, Jeffrey GP, et al. Lamivudine plus low-dose hepatitis B immunoglobulin to prevent recurrent hepatitis B following liver transplantation. Gastroenterology 2007;132:931-937.

11. Jiang L, Yan L, Li B, Wen T, Zhao J, Jiang L, et al. Prophylaxis against hepatitis B recurrence posttransplantation using lamivudine and individualized low-dose hepatitis B immunoglobulin. Am J Transplant 2010;10:1861-1869.

12. Akyildiz M, Karasu Z, Zeytunlu M, Aydin U, Ozacar T, Kilic M. Adefovir dipivoxil therapy in liver transplant recipients for recurrence of hepatitis B virus infection despite lamivudine plus hepatitis B immunoglobulin prophylaxis. J Gastroenterol Hepatol 2007;22:2130-2134.

13. Wang $\mathrm{P}$, Tam $\mathrm{N}$, Wang $\mathrm{H}$, Zheng $\mathrm{H}$, Chen $\mathrm{P}, \mathrm{Wu} \mathrm{L}$, et al. Is hepatitis B immunoglobulin necessary in prophylaxis of hepatitis B recurrence after liver transplantation? A meta-analysis. PLoS One 2014;9:e104480.

14. Moon DB, Lee SG, Hwang S, Kim KH, Ahn CS, Ha TY, et al. Toward more than 400 liver transplantations a year at a single center. Transplant Proc 2013;45:1937-1941.

15. Hwang S, Ahn CS, Kim KH, Moon DB, Ha TY, Song GW, et al. A cross-sectional analysis of long-term immunosuppressive regimens after liver transplantation at Asan Medical Center: increased preference for mycophenolate mofetil. Ann Hepatobiliary Pancreat Surg 2018;22:19-26. 